\title{
Unlocking the lipid labyrinth
}

\author{
Progress in understanding the functions of individual lipids has lagged behind that for other bioactive \\ molecules, but recent technologies that enable the monitoring of individual lipids provide hope.
}

Arguably the most important function of lipids is as the structural basis of membranes, a role for which they are well known. However, the idea that lipids are bioactive molecules has accelerated the recognition of lipids both as critical components in signal transduction and as therapeutic targets for disease. In this themed issue, we highlight recent advances in understanding the structural and signaling functions of lipids, with a particular focus on the development of tools for studying these enigmatic compounds and on the progress in targeting them for disease treatment.

Johann Ludwig Wilhelm Thudichum, considered the father of neurochemistry, named 'sphingolipids' after the Greek sphinx because of the mysterious nature of this class of lipids. As chemical entities, lipids have faced obstacles in being recognized as critical cellular components, in part because of the difficulty of studying such amphiphilic molecules. Further, their ability to form soaps and related micelles has meant that they are much better known for their presence in membranes than for any other bioactivity they might have in the cell. Research on model membranes has established a strong foundation for understanding lipid structure-function relationships but the manipulation of native lipids in vivo remains a formidable challenge.

As well as being an important architectural component of cells, lipid membranes act as mediators of protein-protein and proteinlipid interactions by virtue of their ability to concentrate components that would otherwise not coexist or be localized together within the large cell volume. Indeed, most signaling pathways originate on or around membranes. One of the lipid-based signaling pathways involves the second messengers diacylglycerol and inositol-1,4,5-triphosphate, which are generated upon stimulation of a number of membrane receptors. As the source of these signals, phosphatidylinositol 4,5-bisphosphate $\left(\operatorname{PtdIns}(4,5) \mathrm{P}_{2}\right)$ serves as an intermediate in this pathway. Although they are present at only extremely low levels in cells, PtdIns $(4,5)$ $\mathrm{P}_{2}$ and six other membrane-associated phosphoinositide (PI) lipids are involved in a number of signaling pathways, including membrane trafficking, cell growth and survival. In a review article, Kutateladze (p. 507) focuses on the mechanism of membrane binding and the molecular basis for the binding of PI lipids by specific PI-binding domains in signaling effectors. Because of PI lipids' important roles in signaling, PI detection and manipulation techniques are highly desirable. PI-binding domains in various proteins have proven extremely useful as tools for defining PI localizations among the various cellular membranes.

Lipids also play intracellular roles, and a number of these bioactive lipids have been linked directly to obesity, inflammation and diabetes and indirectly to numerous other diseases, such as cancer. Disease-relevant lipids such as the eicosanoids and the ceramide-derived sphingolipids are among the most highly characterized bioactive lipids. In particular, the sphingolipid sphingosine1-phosphate (S1P) maintains calcium homeostasis, regulates transcription through inhibition of nuclear histone deacetylase activities and serves as a natural ligand for $\mathrm{G}$ protein-coupled receptors, and its misregulation is involved in diseases such as macular degeneration and cancer. Fryst and Saba review the progress in understanding the role of sphingosine-1-phosphate in disease ( $\mathrm{p}$. 489). Much of the knowledge of S1P function was gained by overexpressing or knocking out the biosynthetic genes involved in S1P formation or by characterizing disease-linked metabolic genes. Despite the obvious distance of lipids from the genome, this type of genetic strategy has been the favored way of gaining insight into a lipid's function. Although this has provided a vast understanding of the biosynthetic enzymes involved in lipid metabolism, more direct methods of analysis are needed.

The issues that complicate the study of lipid function also arise in designing strategies to defend against disease due to their misregulation. The best strategy is often to target their biosynthetic and metabolic pathways and the receptors to which they bind, as outlined by Evans and Hutchinson (Commentary, p. 476). However, altering lipid biosynthesis or metabolic enzymes affects numerous lipids, and targeting a biosynthetic enzyme that is already subject to feed-forward or feedback regulation could have undesired consequences.

By simplifying the means to detect and monitor lipids, chemical biologists are contributing in key ways to the goal of understanding the physical and chemical bases of lipid function. Two pieces in this issue describe a variety of recently developed tools that will allow scientists to follow and manipulate lipids, lipid-modified proteins and lipid-binding proteins in vitro and in living cells. Just as green fluorescent protein has enabled monitoring the movements of proteins within cells, chemistries now exist to fluorescently tag lipids in vivo. Schultz (Commentary, p. 473) outlines the promise of chemical tools in quantifying individual lipids and determining their localizations as well as manipulating lipid levels in specific and timely ways. Hannoush and Sun (Review, p. 498) comprehensively describe the existing chemical tools designed to detect protein lipid modifications. Protein modification and regulation by prenylation or fatty acylation is common among signaling proteins, including the attractive therapeutic drug targets $\mathrm{H}$ - and $\mathrm{N}-\mathrm{R}$ as and Src.

Advances in mass spectrometry over the past decade have spawned the field of lipidomics, which has revealed the existence of nearly 100,000 lipid species throughout biology. The Lipid MAPS Consortium (http://lipidmaps.org/) has catalogued approximately 22,000 of these. Different species of lipids clearly arise from modification by a much smaller number of enzymes, calling into question whether knocking down individual biosynthetic or metabolic genes to understand the function of individual lipids is a valid approach.

Despite the remarkable progress highlighted in these articles, the tagging of lipids in cells and in vivo is still in its infancy, and more lipid derivatives that can be tagged are needed. As well, other challenges exist where chemical tools may make a difference. For instance, PI-binding domains used for detection are not useful for determining the spatial organization of lipids. Also, there is much interest in moving beyond the qualitative evaluations of lipid localization and function to more quantitative, mechanistic questions. The recognition of lipids as bioactive molecules and the development of chemical tools has opened up the fields of signaling, lipidomics and disease treatment, but significantly more progress must yet be made in order to uncover all of the mysteries that lipids hold. 